\title{
Differential Role of Mitogen-Activated Protein Kinase in Three Distinct Phases of Memory for Sensitization in Aplysia
}

\author{
Shiv K. Sharma, ${ }^{1}$ Carolyn M. Sherff, ${ }^{1}$ Justin Shobe, ${ }^{1}$ Martha W. Bagnall, ${ }^{1}$ Michael A. Sutton, ${ }^{1}$ and Thomas J. Carew ${ }^{1,2}$ \\ ${ }^{1}$ Department of Neurobiology and Behavior and ${ }^{2}$ Center for the Neurobiology of Learning and Memory, University of California, Irvine, Irvine, California \\ 92697
}

\begin{abstract}
The mitogen-activated protein kinase (MAPK) pathway has been implicated recently in synaptic plasticity and memory. Here we used tail shock-induced sensitization of the tail-elicited siphon withdrawal reflex in Aplysia to examine the role of MAPK in three different phases of memory. We show that a specific pattern of serotonin (5-HT) application that produces intermediate-term and long-term synaptic facilitation (ITF and LTF, respectively) of the sensory-motor (SN-MN) synapses in Aplysia leads to sustained activation of extracellular signal-regulated kinase in the ventrocaudal cluster sensory neurons (SNs), which include the tail SNs. Furthermore, repeated tail shocks that induce intermediate-term and long-term memory (ITM and LTM, respectively) for sensitization also lead to sustained MAPK activation in the SNs. Given these results, we next examined the requirement of MAPK activity in (1) SN-MN synaptic facilitation and (2) memory for sensitization in Aplysia, by inhibiting MEK, the upstream kinase that phosphorylates and activates MAPK. In cellular experiments, we show that MAPK activity is required for ITF of tail SN-tail MN synapses, and, in parallel behavioral experiments, we show that ITM requires MAPK activity for its induction but not its expression. In contrast, short-term memory for sensitization does not require MAPK activity. Finally, 5-HT-induced LTF has been shown previously to require MAPK activity. Here we show that LTM for sensitization also requires MAPK activity. These results provide evidence that MAPK plays important roles specifically in long-lasting phases of synaptic plasticity and memory.
\end{abstract}

Key words: synaptic facilitation; sensitization; learning; memory; MAP kinase; ERK

\section{Introduction}

Recent studies have implicated the extracellular signal-regulated kinase [mitogen-activated protein kinase (MAPK)] pathway as a key regulator of long-term synaptic plasticity and long-term memory (LTM) (for review, see Adams and Sweatt, 2002). For example, in the hippocampus, a requirement for MAPK activity has been demonstrated for long-term potentiation (LTP) (English and Sweatt, 1997; Atkins et al., 1998; Impey et al., 1998; Winder et al., 1999; Wu et al., 1999), and, in Hermissenda, stimuli that produce long-term neuronal plasticity lead to activation of MAPK (Crow et al., 1998). A requirement of MAPK activity has also been shown for long-term memory in a variety of learning tasks (Atkins et al., 1998; Berman et al., 1998; Blum et al., 1999; Schafe et al., 1999; Selcher et al., 1999; Walz et al., 1999, 2000). Thus, MAPK plays important roles in the formation of long-term memories, raising an interesting question: what is the role of MAPK in the induction of memories falling within other time domains?

Although it is common to distinguish short-term memory (STM) from LTM, it is now also possible to distinguish unique intermediate phases of synaptic plasticity (Ghirardi et al., 1995; Crow et al., 1997; Winder et al., 1998; Sutton and Carew, 2000) and memory (Rosenzweig et al., 1993; DeZazzo and Tully, 1995; Menzel, 2001; Sutton et al., 2001). For example, synaptic facilita-

Received Nov. 27, 2001; revised Jan. 29, 2003; accepted Feb. 3, 2003.

This work was supported by National Science Foundation Grant IBN-0049013 (T.J.C.). We thank A. Purcell for her comments on a previous version of this manuscript.

Correspondence should be addressed to Thomas J. Carew at the above address. E-mail: tcarew@uci.edu. Copyright $\odot 2003$ Society for Neuroscience $\quad$ 0270-6474/03/233899-09\$15.00/0 tion of sensory-motor (SN-MN) synapses in Aplysia, a cellular model of behavioral sensitization, exists in at least three mechanistically distinct temporal phases (Montarolo et al., 1986; Ghirardi et al., 1995; Mauelshagen et al., 1996; Sutton and Carew, 2000). A single pulse of serotonin (5-HT), a modulatory neurotransmitter released during sensitization training (Marinesco and Carew, 2002; S. Marinesco and T. J. Carew, unpublished observations; see also Levenson et al., 1999), produces short-term facilitation (STF) of SN-MN synapses ( $<30 \mathrm{~min}$ ), whereas five pulses produce two temporally distinct phases: intermediateterm facilitation (ITF) ( $>90 \mathrm{~min}$ ) and long-term facilitation (LTF) (>24 hr) (Montarolo et al., 1986; Ghirardi et al., 1995; Sutton and Carew, 2000). Similarly, a single tail shock produces STM (<30 min) for sensitization (Goldsmith and Byrne, 1993; Sutton et al., 2001), whereas repeated shocks produce both intermediate-term memory (ITM) (lasting >90 min) and LTM ( $>24$ hr) (Castellucci et al., 1989; Levenson et al., 2000; Sutton et al., 2001).

In Aplysia, MAPK plays a critical role in the induction of LTF. Five pulses of 5-HT activate MAPK (Michael et al., 1998) and induce its translocation to the sensory neuron nucleus (Martin et al., 1997), and inhibition of MAPK blocks the induction of LTF in cultured SN-MN synapses (Martin et al., 1997). Because MAPK plays a critical role in LTF, one would predict that it may also be important for LTM. Moreover, the fact that multiple phases of synaptic plasticity and memory can be distinguished in this system allows for the additional opportunity to examine the role of MAPK not only in LTM but in ITF and ITM as well.

We investigated the role of MAPK in three temporally and mechanistically distinct phases of memory for sensitization in 
Aplysia: STM, ITM, and LTM. We find that five pulses of 5-HT, as well as five tail shocks, produce a sustained activation of MAPK in ventrocaudal (VC) cluster SNs. Moreover, blocking MAPK activity blocks ITF induced by repeated tail nerve shocks. Examining memory, we find that ITM and LTM require MAPK activity, whereas STM does not. These results show that the MAPK signaling cascade plays a critical role in the induction of long-lasting phases of both synaptic plasticity and memory in Aplysia.

Parts of this paper have been published previously in abstract form (Sharma et al., 2002).

\section{Materials and Methods}

MAP kinase activation. For experiments examining MAPK activation by five spaced pulses of 5-HT, wild-caught Aplysia californica (250-400 gm; supplied by Marinus, Long Beach, CA) were anesthetized by injecting isotonic $\mathrm{MgCl}_{2}(\sim 100 \mathrm{ml} / 100$ gm body weight), and pleural-pedal ganglia were removed. The ganglia were pinned in a dish coated with Sylgard and desheathed in 50:50 $\mathrm{MgCl}_{2} /$ artificial seawater (ASW) (containing in mM: $460 \mathrm{NaCl}, 55 \mathrm{MgCl}_{2}, 11 \mathrm{CaCl}_{2} 10 \mathrm{KCl}$, and 10 Tris, $\mathrm{pH}$ 7.6) to expose the neurons. After desheathing, the ganglia were washed with ASW for 15 min to remove $\mathrm{MgCl}_{2}$. Five pulses of $50 \mu \mathrm{M} 5$-HT were applied to the pleural-pedal ganglia by perfusion (dish volume, $\sim 4 \mathrm{ml}$; flow rate, $\sim 5$ $\mathrm{ml} / \mathrm{min}$ ). Each 5 -HT pulse lasted for $5 \mathrm{~min}$ with $15 \mathrm{~min}$ wash with ASW between pulses. Entire VC cluster SNs, of which the tail SNs are a component, were excised either immediately $(0 \mathrm{hr})$ or 1,3 , or $20 \mathrm{hr}$ after the last pulse of 5-HT (for $0 \mathrm{hr}$ time point, the SN clusters were excised in 5-HT and lysed within $3 \mathrm{~min}$, whereas for 1, 3, or $20 \mathrm{hr}$ time points, 5-HT was washed out by continuous perfusion with ASW for $15 \mathrm{~min}$ and ganglia were incubated at $15^{\circ} \mathrm{C}$ before harvesting the SN clusters). For experiments examining the effect of a single pulse of 5-HT on MAPK activation, ganglia were exposed to $50 \mu \mathrm{M} 5-\mathrm{HT}$ for $5 \mathrm{~min}$, and SN clusters were excised in 5-HT and lysed within $3 \mathrm{~min}$. For experiments examining the effect of MEK (the upstream kinase that phosphorylates and activates MAPK) inhibitor (see Fig. $4 B$ ), five spaced pulses of 5-HT were applied to the pleural-pedal ganglia. The last pulse of 5-HT was washed out by continuous perfusion with ASW for $15 \mathrm{~min}$, the ganglia remained at room temperature for another $15 \mathrm{~min}$, and then MEK inhibitor (U0126, $20 \mu \mathrm{M})$ or its inactive analog (U0124, $20 \mu \mathrm{M}$ ) was applied for 60 min, immediately after which, the SN clusters were excised. MEK1/2 inhibitor (U0126; active) and its inactive analog (U0124; inactive) were obtained from Calbiochem (La Jolla, CA) and dissolved in DMSO (20 mM stock) and diluted in ASW to $20 \mu \mathrm{m}$ final concentration just before use. For experiments examining the activation of MAPK by repeated tail shocks, five shocks (AC, 1.5 sec duration) with an intertrial interval (ITI) of $10 \mathrm{~min}$ were delivered to one side of the tail through a hand-held bipolar electrode (shock area, $9 \mathrm{~mm}$ ). Current flow through the skin was achieved through a salt bridge between the two poles of the electrode. The nominal current across the electrode was $100 \mathrm{~mA}$, although much of this current is shunted by the seawater. Animals were anesthetized by injection of isotonic $\mathrm{MgCl}_{2}$, ganglia were removed, and SN clusters were excised. Anesthetization of animals was timed so that the total time between the last shock and lysis of the SN clusters was 1, 3, or $22 \mathrm{hr}$.

After excision, the SN clusters were centrifuged in a microfuge for 15 sec, supernatant was removed, and cells were immediately lysed in the lysis buffer [50 mm Tris-Cl, pH 7.5, 150 mм NaCl, 1 mм EDTA, 1 mм EGTA, $50 \mathrm{~mm}$ sodium fluoride, $1 \mathrm{~mm}$ sodium orthovanadate, $10 \mathrm{~mm}$ sodium $\beta$-glycerophosphate, $4 \mathrm{~mm}$ para-nitrophenylphosphate, $2 \%$ SDS, and a protease inhibitor mixture (one tablet of Roche Diagnostics (Mannheim, Germany) protease inhibitor mixture/25 ml)], boiled for 2 min, centrifuged at top speed in a microfuge for 5 min to remove any insoluble material, and stored at $-80^{\circ} \mathrm{C}$ until use.

Proteins were resolved on $11 \%$ SDS-polyacrylamide gels (Laemmli, 1970) and electrophoretically transferred to Hybond C membranes (Amersham Biosciences, Piscataway, NJ). The blots were washed (three times for $5 \mathrm{~min}$ each) with Tris-buffered saline containing $0.1 \%$ Tween 20 (TBST) (25 mm Tris-Cl, $137 \mathrm{~mm} \mathrm{NaCl}, 2.7 \mathrm{~mm} \mathrm{KCl,} \mathrm{pH} \mathrm{7.5,} \mathrm{and} \mathrm{0.1 \%}$ Tween 20). A 5\% solution of BSA (Fraction V; Sigma, St. Louis, MO) in TBST was used as a blocking agent. Primary (anti-phospho-p44/p42
MAPK and phospho-independent p44/p42 MAPK) and secondary (HRP-conjugated anti-rabbit) antibodies (all from Cell Signaling Technology, Beverly, MA) were diluted in TBST containing 5\% BSA. Sodium fluoride $(20 \mathrm{~mm})$ and sodium orthovanadate $(2 \mathrm{~mm})$ were included in BSA-containing solutions to inhibit the possible contaminant phosphatases (Sharma and Carew, 2002). Incubation with the blocking buffer was performed for $1 \mathrm{hr}$ at room temperature, after which blots were incubated with the primary antibody overnight at $4^{\circ} \mathrm{C}$. After washing with TBST (three times for $5 \mathrm{~min}$ each), blots were incubated with the secondary antibody for $1 \mathrm{hr}$ at room temperature. Blots were again washed with TBST (three times for 5 min each), and signal was detected using the ECL reagent (Amersham Biosciences). To obtain the total MAPK signal in each sample, the blots were stripped after obtaining the signal with the phospho-MAPK antibody and reprobed with the MAPK antibody that is independent of the phosphorylation state of the protein. Stripping was performed by incubating the blots in stripping buffer $(62.5 \mathrm{~mm}$ Tris- $\mathrm{Cl}$, pH 6.8, $100 \mathrm{~mm} \beta$-mercaptoethanol, and $2 \%$ SDS) at $65^{\circ} \mathrm{C}$ for $45 \mathrm{~min}$ and was confirmed by probing the blots without the primary antibody. Exposure of the film was kept in the linear range, and band intensity was quantified using NIH Image software (National Institutes of Health, Bethesda, MD). The phospho-MAPK signal was normalized to the total MAPK signal in each sample (Patterson et al., 2001; Mazzucchelli et al., 2002; Purcell et al., 2003). Although the phospho-MAPK and phosphoindependent MAPK antibodies recognize well separated p44 and p42 in mammalian systems, consistent with previous observations (Michael et al., 1998; Chin et al., 2002; Purcell et al., 2003), we routinely observed a single band in Aplysia SN extracts. Occasionally, however, we observed two bands that run close together.

Electrophysiology. Animals were anesthetized by injection of isotonic $\mathrm{MgCl}_{2}$ as described above. One pair of pleural-pedal and the paired cerebral ganglia were removed and pinned in a Sylgard-coated recording dish. The pleural-pedal ganglia were then desheathed in $\mathrm{ASW} / \mathrm{MgCl}_{2}$ (50:50) to prevent synaptic transmission during dissection. The tail nerve (P9) was drawn up into a suction electrode. The $\mathrm{MgCl}_{2}$ was then washed out by perfusing ASW for $15 \mathrm{~min}$. Microelectrode recordings were made from monosynaptically connected tail SNs and tail MNs. The MN was hyperpolarized to $-70 \mathrm{mV}$ to prevent action potentials.

Two pretests ( 15 min apart) of the $\mathrm{SN}-\mathrm{MN}$ synapses were made by eliciting a single action potential in the $\mathrm{SN}$ (with a $5 \mathrm{msec}$ depolarizing current pulse) and recording the evoked EPSP in the MN. Only stable synapses, those with baseline EPSP amplitudes within $20 \%$ of the mean, were used. After the second pretest, ganglia were perfused with $20 \mathrm{ml}$ of either U0126 or U0124 (20 $\mu \mathrm{M}$; dish volume, $\sim 4 \mathrm{ml}$; flow rate, $\sim 5 \mathrm{ml} /$ $\mathrm{min})$. The perfusion was stopped, and the ganglia were incubated for 30 $\mathrm{min}$. At the end of incubation, a third pretest measurement was performed (as above) to determine whether either drug affected baseline synaptic transmission. After another 15 min interval, five spaced shocks ( $5 \mathrm{msec}$ pulses at $40 \mathrm{~Hz}$ for $2 \mathrm{sec}$ ) were delivered to the tail nerve (P9) with an ITI of $15 \mathrm{~min}$. Another $20 \mathrm{ml}$ of drug solution was perfused after the fourth shock. Intermediate-term facilitation was measured by testing (as above) the same synapse at 30 and $45 \mathrm{~min}$ after the last shock. Baseline EPSP was determined by the average of the pretests.

Behavioral procedures. We used a reduced preparation of Aplysia (see Fig. 3A) (for details, see Sutton et al., 2001) for the behavioral experiments. The animals were anesthetized by injecting isotonic $\mathrm{MgCl}_{2}$. The tail and mantle were surgically removed while keeping the CNS circuitry (ring ganglia and abdominal ganglion) and peripheral connections intact. The tail and siphon were continually perfused with cooled tank seawater $\left(15^{\circ} \mathrm{C}\right.$; Instant Ocean; Aquarium Systems, Mentor, $\left.\mathrm{OH}\right)$ to keep them inflated throughout the experiment. The siphon artery was cannulated with SILASTIC tubing (inner diameter, 0.25 inches) and perfused at $\sim 5 \mathrm{ml} / \mathrm{min}$, whereas the tail was perfused at $\sim 0.5 \mathrm{ml} / \mathrm{min}$ through a 22 gauge needle inserted into the medial aspect of the tail. The tail and mantle were pinned to the Sylgard-coated floor of a chamber containing circulating tank seawater $\left(15^{\circ} \mathrm{C}\right.$ ), and the ring ganglia (comprising the cerebral and two paired pleural-pedal ganglia) were pinned in a separate Sylgard-coated chamber and perfused continuously (except during drug incubations, which were in static bath) at room temperature with ASW. The ring ganglia (with both pleural-pedal ganglia desheathed) were iso- 
lated in this separate subchamber and perfused independently of the rest of the preparation. The drug treatments were restricted to the ring ganglia subchamber. The connections to the tail (via the P9 nerves) and abdominal ganglion (via the pleural-abdominal connectives) exited the subchamber through small slits that were sealed with Vaseline. Preparations were allowed at least $60 \mathrm{~min}$ to recover before pretest measurements.

Before training, three to four pretests with an interstimulus interval of 15 min were conducted to measure baseline tail-elicited siphon withdrawal (T-SW) duration. In each of these tests, the right or left side of the tail $(\sim 1 \mathrm{~cm}$ anterior from its tip and midway between the lateral and medial margins) was stimulated with a brief water jet $(8 \mathrm{~Hz}, 0.5 \mathrm{sec}$ duration). Similar results were obtained for right and left sides. For training, AC tail shocks with an ITI of 10 min were delivered to the top of the tail on the side used for pretest measurements. Tests of T-SW after training (elicited in the same manner as the pretests described above) were conducted at different times as required depending on the phase of memory under examination.

The drug incubations were performed in ganglia subchambers, and baths were exchanged manually. For experiments examining ITM for sensitization, U0126 or U0124 (20 $\mu \mathrm{M})$ was applied to the ganglia subchamber (five bath exchanges) after the two pretests and incubation was performed for $30 \mathrm{~min}$. Two additional pretests were performed during this time. The drug baths were exchanged with fresh drug solutions (2 bath exchanges), and the animals were trained by delivering five spaced shocks to the tail. The drug baths were again exchanged with fresh drug solutions (two bath exchanges) at the end of the training, and measurements of T-SW were performed every $15 \mathrm{~min}$ until $75 \mathrm{~min}$ after training. For long-term sensitization experiments, the inhibitor was applied after baseline measurements, bath exchanges with drug solutions and training were similar to the ITM experiment, except that drug incubations were performed for $1 \mathrm{hr}$ after training, after which they were washed out by continuous perfusion with ASW for 15 min and preparations were perfused with cooled seawater overnight. The measurements were performed at $18 \mathrm{hr}$ after training. For experiments examining STM for sensitization, inhibitor applications were similar to the ITM experiment, and a single tail shock, which was delivered at the time of last shock in the ITM experiment, was used as training. Tests were performed at 5, 10, 15, and 45 min thereafter. For experiments examining the requirement of MAPK activity specifically in the induction of ITM, inhibitor application and training were identical to the ITM experiment described above, except that the drugs were washed off immediately after training by continuous perfusion with ASW. For experiments examining the effect of MEK inhibitor on the expression of ITM, the inhibitor was applied after 30 min posttraining test, and measurements of T-SW were taken every 30 $\mathrm{min}$. The drug baths were exchanged with fresh drug solutions at 60 and $100 \mathrm{~min}$ after training (to be consistent with the ITM experiment described above).

Data analysis. Duration of T-SW was measured by an observer who was blind to both training and drug treatment. The duration of T-SW was quantified as the elapsed time from stimulus onset to the initial relaxation of the siphon from the contracted position (Sutton et al., 2001). Baseline duration of T-SW was determined by the average of the pretests. Statistical analyses of synaptic and behavioral results were performed with either $t$ tests or repeated-measures ANOVA, followed by factorial ANOVA when appropriate. The Fisher's least significant difference post hoc test was used after factorial ANOVA. For MAPK activation, $t$ tests were performed on raw phospho-MAPK/total MAPK ratios between experimental and control groups.

\section{Results}

Repeated pulses of 5-HT and repeated tail shocks induce sustained activation of MAPK in the VC cluster sensory neurons

It has been shown previously that MAPK is activated in sensory neurons in response to five spaced pulses of 5-HT (Michael et al., 1998; Purcell et al., 2003). However, the time course of MAPK activation is not known. To examine the temporal profile of

\section{A. SEROTONIN}

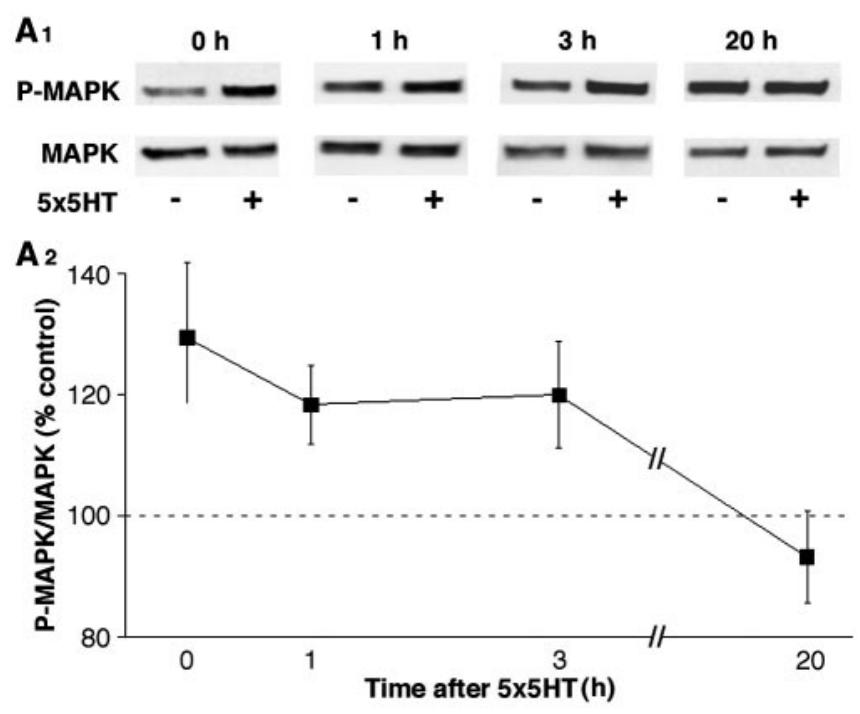

\section{B. TAIL SHOCK}

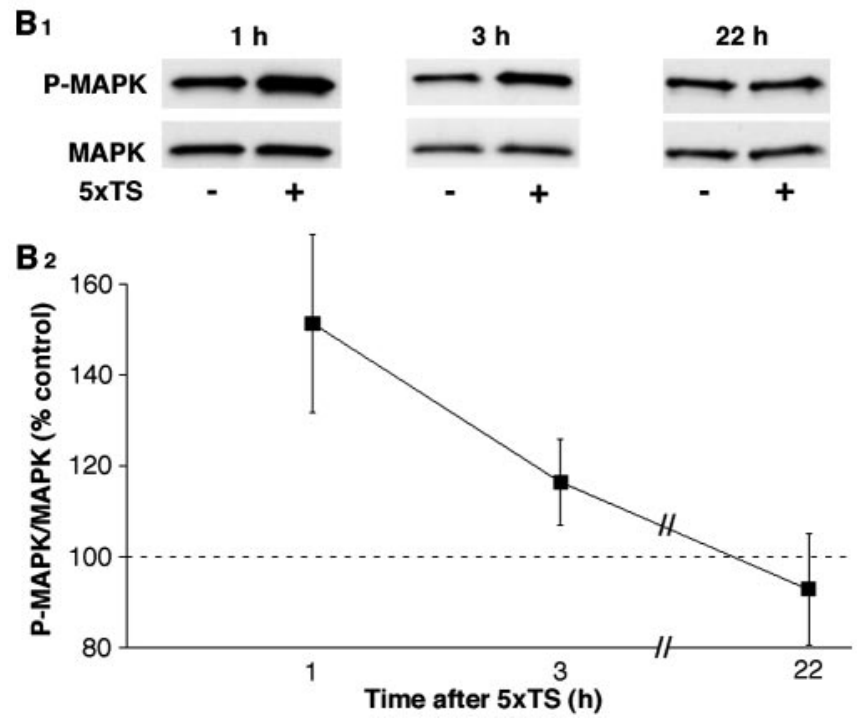

Figure 1. Time course of MAP kinase activation. $A$, Time course of MAPK activation by five spaced pulses of 5 -HT. Pleural-pedal ganglia were treated with five pulses of 5-HT $(5 \times 5 \mathrm{HT})$, SN clusters were excised either immediately $(0 \mathrm{~h})$ or $1(1 \mathrm{~h}), 3(3 \mathrm{~h})$, or $20(20 \mathrm{~h}) \mathrm{hr}$ after the last pulse of 5-HT, and MAPK activation was examined using phospho-MAPK and phosphoindependent MAPK antibodies. $A_{1}$, Sample blots with phospho-MAPK and total MAPK antibodies. $A_{2}$, Summary of time course of MAPK activation by five spaced pulses of 5-HT. Data are presented as mean \pm SEM (percentage of control; $n=12$ per group, each time point). The 5 -HT-treated samples are normalized to their paired ASW controls at each time point. $B$, Time course of MAPK activation by five spaced tail shocks. Animals were given five spaced shocks $(5 \times$ TS) to one side of the tail, SN clusters were excised at the indicated times, and MAPK activation was examined as described above. $B_{1}$, Sample blots with phospho-MAPK and total MAPK antibodies. $B_{2}$, Summary of time course of MAPK activation by five spaced tail shocks. Data are presented as mean \pm SEM [percentage of control; $n=8(1 \mathrm{hr}), 7(3 \mathrm{hr})$, and $6(22 \mathrm{hr})$ ]. The shocked side samples are normalized to their paired nonshocked control side samples at each time point.

MAPK activation, desheathed pleural-pedal ganglia were exposed to five pulses of 5-HT, and SN clusters were excised either immediately or 1, 3, or $20 \mathrm{hr}$ after the last pulse of 5-HT and assayed for MAPK activation using phospho-MAPK and phospho-independent MAPK antibodies. These time points were chosen to examine the MAPK activation over the short-term, 
intermediate-term, and long-term phases of synaptic facilitation. As shown in Figure $1, A_{1}$ and $A_{2}$, MAPK was activated immediately after the application of five pulses of 5-HT, remained activated for at least $1 \mathrm{hr}$ thereafter, and returned to basal levels by the $20 \mathrm{hr}$ time point $\left(0 \mathrm{hr}, 129.36 \pm 12.37 \%, t_{(11)}=2.25 ; 1 \mathrm{hr}\right.$, $118.29 \pm 6.60 \%, t_{(11)}=2.82 ; p<0.05$ for both time points; $3 \mathrm{hr}$, $119.93 \pm 8.80 \%, t_{(11)}=2.00 ; 20 \mathrm{hr}, 93.17 \pm 7.64 \%, t_{(11)}=-1.18$; both NS). Although MAPK activation $3 \mathrm{hr}$ after five pulses of 5-HT was no longer statistically significant, it still remained above baseline. Thus, five spaced pulses of 5-HT lead to sustained MAPK activation in the SNs.

Because 5-HT is known to be released onto the SNs in response to tail nerve shock and tail shock (Marinesco and Carew, 2002; Marinesco and Carew, unpublished observations), we next examined whether repeated shocks to the animal's tail (stimuli used for behavioral studies, see below) also lead to sustained MAPK activation. The animals were given five spaced tail shocks, and MAPK activation was examined in the SNs 1, 3, or $22 \mathrm{hr}$ after the last shock using phospho-MAPK and phospho-independent MAPK antibodies. As shown in Figure $1, B_{1}$ and $B_{2}$, MAPK was significantly activated in the SNs after $1 \mathrm{hr}$ and returned to basal levels by $22 \mathrm{hr}$ after the last shock $\left(1 \mathrm{hr}, 151.37 \pm 19.63 \%, t_{(7)}=\right.$ $2.90, p<0.05 ; 3 \mathrm{hr}, 116.46 \pm 9.51 \%, t_{(6)}=1.53$, NS; $22 \mathrm{hr}$, $\left.92.83 \pm 12.20 \%, t_{(5)}=-0.11, \mathrm{NS}\right)$. Similar to observations with 5-HT (Fig. 1A), although MAPK activation $3 \mathrm{hr}$ after the last shock was no longer statistically significant, it was above the baseline. Thus, both repeated pulses of 5-HT and behaviorally relevant sensitizing stimuli (tail shocks) lead to a qualitatively similar prolonged time course of MAPK activation in the SNs.

\section{MAP kinase activity is required for intermediate-term facilitation of tail SN-tail MN synapses}

It has been shown previously that five spaced pulses of 5-HT induce ITF lasting up to $3 \mathrm{hr}$ at the SN-MN synapses (Ghirardi et al., 1995; Mauelshagen et al., 1996; Sutton and Carew, 2000) and that a comparable pattern of tail shocks induce ITM for sensitization in the same time domain (Sutton et al., 2001). Thus, our observation that five pulses of 5-HT and five repeated tail shocks induce sustained activation of MAPK in the intermediate-term time domain suggested that it might play a role in ITF at tail $\mathrm{SN}-\mathrm{MN}$ synapses. To keep our cellular experiments as behaviorally relevant as possible (see memory experiments below), we used tail nerve shock to induce ITF. As mentioned above, we know that such nerve shock (as well as tail shock) releases 5-HT onto the SNs (Marinesco and Carew, 2002; Marinesco and Carew, unpublished observations), and it is already well established that direct pulses of 5-HT also induce ITF. We examined the role of MAPK in ITF using an inhibitor (U0126) of MEK1/2, a kinase that acts upstream of MAPK and activates it. U0126 is a specific MEK1/2 inhibitor (DeSilva et al., 1998) and has been shown to block MAPK activation in Aplysia (Chin et al., 2002) (also see below). The MEK inhibitor (U0126) or its inactive ana$\log$ (U0124) was applied $45 \mathrm{~min}$ before tail nerve shock and was present during the course of nerve shocks and throughout the testing period. The results shown in Figure 2 demonstrate that, whereas ITF was observed when the preparations were treated with U0124 (inactive), ITF was abolished in the preparations treated with U0126 (active). Thus, there was a significant difference in ITF between the active and inactive groups ( $30 \mathrm{~min}, t_{(7)}=$ $-2.72 ; 45$ min, $t_{(6)}=-2.46 ; p<0.05$ both time points). Consistent with previous observations using a different MEK inhibitor (PD098059) (Martin et al., 1997), U0126 did not have any effect on basal synaptic transmission $\left(t_{(3)}=0.15 ; \mathrm{NS}\right)$. Thus, our results
A
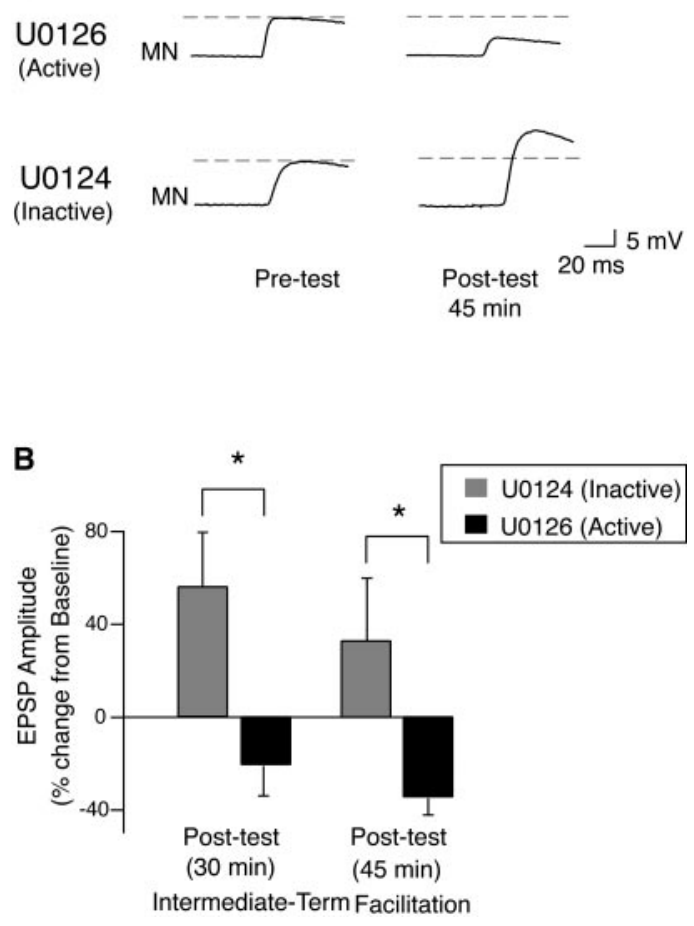

Figure 2. MAP kinase activity is required for intermediate-term facilitation of tail $\mathrm{SN}$-tail MN synapses. Representative traces $(A)$ and summary data $(B)$ of EPSP amplitudes showing that ITF was induced by five tail nerve shocks in preparations treated with the inactive analog of MEK inhibitor (U0124), but ITF was completely blocked in preparations treated with the MEK inhibitor (U0126). U0126 or U0124 was applied $45 \mathrm{~min}$ before tail nerve shock and was present during the course of nerve shocks and throughout the testing period. Data are presented as mean \pm SEM (percentage of change from baseline; $30 \mathrm{~min}, n=4$ and 5, U0126 and U0124, respectively; $45 \mathrm{~min}, n=4$ per group). The dashed lines indicate baseline EPSP amplitudes. ${ }^{*} p<0.05$.

demonstrate that, in addition to LTF (Martin et al., 1997), MAPK activity is required for ITF of tail SN-MN synapses.

\section{MAP kinase activity is required for intermediate-term memory for sensitization in Aplysia}

Facilitation of the SN-MN synapses is thought to contribute to memory for sensitization in Aplysia. Our results revealing a requirement of MAPK for ITF suggested that MAPK activity might also be required for ITM for sensitization. To examine this question, we made use of a reduced preparation of Aplysia (Fig. 3A) (Sutton et al., 2001) in which pharmacological agents can be selectively applied to the ring ganglia while simultaneously measuring T-SW. After obtaining two pretests, U0126 or U0124 was applied to the ring ganglia subchamber $30 \mathrm{~min}$ before training and was present during training and throughout the testing period. As shown in Figure $3 B$, the preparations treated with the MEK inhibitor did not show ITM, whereas the preparations treated with the inactive analog showed significant ITM $\left(F_{(5,25)}=6.12 ; p<\right.$ $0.001)$. Between-group analysis revealed a significant enhancement of T-SW in trained preparations treated with U0124 relative to both trained and nontrained preparations treated with U0126 (15-75 min; all $p<0.05$ ) but no difference between the two groups of U0126-treated preparations (15-75 min; all NS). Importantly, the same course of treatment with U0126 had no effect on baseline T-SW in nontrained control preparations examined in parallel $\left(F_{(5,10)}=0.38\right.$; NS). These results demonstrate that MAPK activity is required for intermediate-term memory for sensitization. 
A

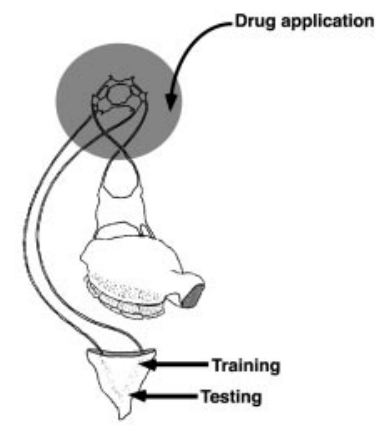

B

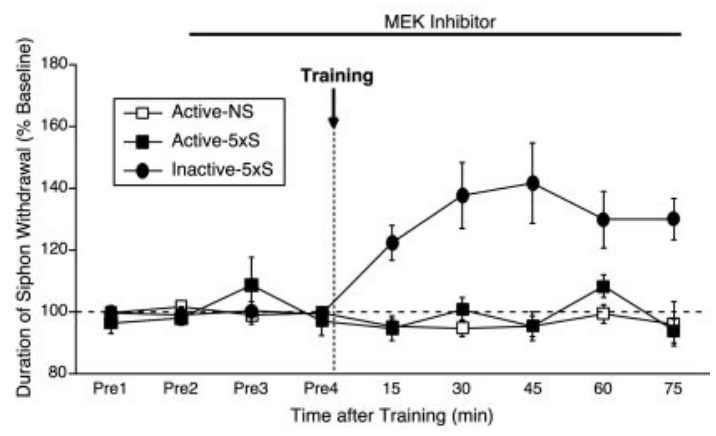

that MAPK activity is required for the induction but not expression of ITM induced by repeated tail shocks.

\section{MAP kinase activity is not required for short-term memory for sensitization in Aplysia}

Having shown that MAPK activity is critical for the induction of ITM, we turned our attention to STM. It is clear from Figures 3 and $4 A$ that, after five tail shocks, there was virtually no memory for sensitization, even at $15 \mathrm{~min}$ after the last shock in animals that were treated with the MEK inhibitor. This raised the possibility that STM also required MAPK activity. To examine this question, we first examined the effect of a single pulse of 5-HT on MAPK activation. As shown in Figure 5 (inset), consistent with previous results (Michael et al., 1998; Purcell et al., 2003), a single pulse of 5 -HT did not activate MAPK $\left(90.76 \pm 6.13 \% ; t_{(22)}=-1.45\right.$; NS $)$. We

Figure 3. MAP kinase activity is required for intermediate-term memory for sensitization. $A$, Schematic diagram of the reduced preparation used for behavioral experiments. In this preparation, pharmacological agents can be selectively applied to the ring ganglia (shaded area). Shocks were administered laterally on the tail at a different site from where the test stimuli were applied (both sites are shown by arrows). B, U0126 (Active) or the inactive analog U0124 (Inactive) was applied to the ring ganglia 30 min before training and was present during training and throughout the testing period. Training was performed using five spaced shocks to the tail $(5 \times S)$. NS, Control preparations not given any shock. Data are expressed as mean \pm SEM duration of T-SW normalized to baseline (Active- $5 \times S, n=6$; Inactive $5 \times S, n=6$; Active-NS, $n=3$ ). In this and subsequent behavioral figures, horizontal dashed line denotes baseline T-SW, and vertical dashed line denotes training.

\section{MAP kinase activity is required for the induction but not expression of intermediate-term memory}

Our results in the preceding section showed that ITM for sensitization requires MAPK activity. We were interested to know whether MAPK activity was required for the processes that produce ITM (induction) and/or the processes that maintain ITM (expression). To examine whether MAPK activity was required for the induction of ITM, we applied the MEK inhibitor $30 \mathrm{~min}$ before training and restricted the application to the training period. As shown in Figure $4 A$, relative to the preparations treated with the inactive analog, ITM was significantly reduced in the preparations treated with the MEK inhibitor $\left(F_{(1,6)}=12.73\right.$; $p<$ $0.05)$, showing that MAPK activity is required for the induction of ITM.

We next examined whether the expression of ITM also requires MAPK activity. To explore this question, it was necessary to ensure that the MEK inhibitor reduced MAPK activation after it was already induced by five spaced pulses of 5-HT. Thirty minutes after pleural-pedal ganglia were exposed to five pulses of 5-HT, one group was exposed to U0126 and the other group was exposed to U0124, and incubation was performed for $60 \mathrm{~min}$. As shown in Figure $4 B$, the samples treated with U0126 showed significantly reduced MAPK activity compared with the samples treated with U0124 $\left(-53.20 \pm 5.76 \%\right.$ change from U0124; $t_{(6)}=$ $-7.21 ; p<0.05)$.

We next examined whether expression of ITM was dependent on MAPK activity. In these experiments, the treatment with MEK inhibitor or its inactive analog began 30 min after training and continued throughout ITM expression. As shown in Figure 4C, five spaced tail shocks induced significant ITM for sensitization (30 min after training) in both groups $\left(t_{(5)}=3.13, p<0.05\right.$ and $t_{(4)}=3.22, p<0.05$, for preparations later treated with U0126 and U0124, respectively), and the magnitude of ITM was comparable between them $\left(t_{(9)}=0.81\right.$; NS). The expression of ITM was maintained over time in preparations treated with either U0126 $\left(30-150 \mathrm{~min} ; F_{(4,20)}=0.66\right.$; NS $)$ or U0124 $\left(30-150 \mathrm{~min} ; F_{(4,16)}=\right.$ 0.85 ; NS). Moreover, between-group comparisons failed to reveal any significant difference in the magnitude of ITM throughout the time of testing $\left(60-150 \mathrm{~min}\right.$; all $F_{(1,9)}<0.99$; NS). These results, together with the ITM induction results (Fig. $4 A$ ), show next examined STM induced by a single tail shock. U0126 or U0124 was applied 70 min before training (to be consistent with the ITM experiments described above in which the last of the five shocks came $70 \mathrm{~min}$ after drug application) and was present throughout the testing period. As shown in Figure 5, significant sensitization was observed in both groups (5-15 min; U0126, $\left.F_{(3,15)}=6.22, p<0.05 ; \mathrm{U} 0124, F_{(3,12)}=3.66, p<0.05\right)$, and STM for sensitization was not different in U0126- and U0124-treated preparations (5-15 min; all $F_{(1,9)}<0.46$; NS). Confirming a previous report (Sutton et al., 2001), memory for sensitization after one tail shock decayed to baseline within $45 \mathrm{~min}$ in both U0126and U0124-treated preparations. Thus, neither the magnitude nor the overall temporal profile of STM after a single shock was affected by inhibiting MEK. These results show that MAPK activity is not required for STM for sensitization in Aplysia. The apparent lack of STM in the presence of MEK inhibitor after multiple training trials (Figs. 3, $4 \mathrm{~A}$ ) is an interesting question that we will address in Discussion.

\section{MAP kinase activity is required for long-term memory for sensitization in Aplysia}

It has been shown previously that MAPK activity is required for long-term facilitation of SN-MN synapses in culture (Martin et al., 1997). However, the role of MAPK in behaviorally expressed long-term memory for sensitization has not been examined in Aplysia. Thus, in a final series of experiments, we examined the role of MAPK in LTM. After taking baseline measurements, U0126 or U0124 was applied $30 \mathrm{~min}$ before training and was present during training and for $1 \mathrm{hr}$ after the training. As shown in Figure 6, in preparations treated with the inactive analog of MEK inhibitor, five spaced tail shocks induced significant LTM for sensitization $18 \mathrm{hr}$ after training $\left(t_{(5)}=2.23\right.$; $\left.p<0.05\right)$. In contrast, preparations treated with the MEK inhibitor did not show LTM $\left(t_{(5)}=1.05\right.$; NS). Importantly, the same course of treatment with U0126 had no effect on baseline T-SW at the long-term test in nontrained control preparations examined in parallel $\left(t_{(4)}=0.25\right.$; NS). Between-group comparisons revealed a significant enhancement of T-SW at the long-term test in U0124treated preparations trained with five tail shocks relative to both trained and nontrained preparations treated with U0126 

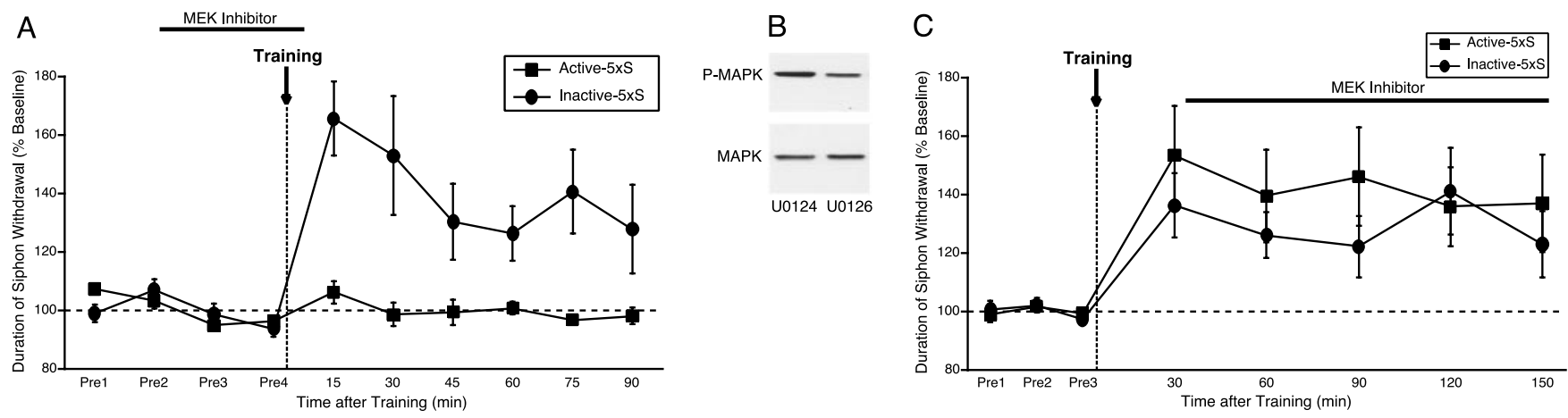

Figure 4. MAP kinase activity is required for the induction but not expression of intermediate-term memory. A, MAPK activity is required for the induction of ITM. The MEK inhibitor U0126 (Active; $n=4$ ) or its inactive analog U0124 (Inactive; $n=4$ ) was applied to the ring ganglia 30 min before training and was present during training but washed off immediately after training. Training was performed using five spaced shocks to the tail $(5 \times S)$. Data are expressed as mean \pm SEM duration of T-SW normalized to baseline. $B$, The MEK inhibitor blocks MAPK activation when applied 30 min after five pulses of 5-HT. Pleural-pedal ganglia were treated with five pulses of 5-HT. U0126 or U0124 was applied 30 min after the last pulse of 5-HT and incubated for 60 min, and MAPK activation was examined in the SNs using phospho-MAPK and phospho-independent MAPK antibodies. Sample blots with phospho-MAPK and total MAPK antibodies are shown. C, MAPK activity is not required for the expression of ITM. U0126 (Active; $n=6$ ) or U0124 (Inactive; $n=5$ ) was applied to the ring ganglia after the 30 min posttraining test and was present throughout testing. Training was performed using five spaced shocks to the tail $(5 \times S)$. Data are expressed as mean \pm SEM duration of T-SW normalized to baseline.

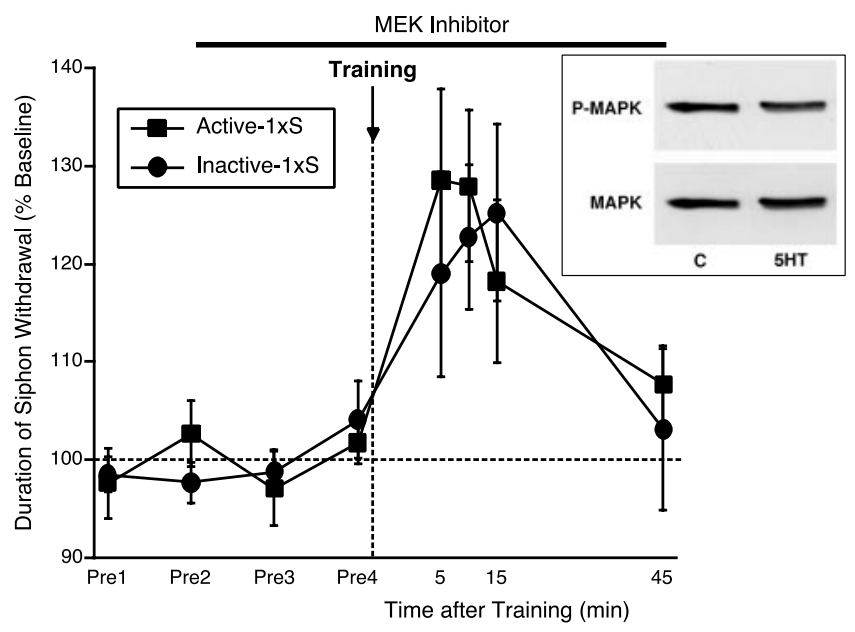

Figure 5. MAP kinase activity is not required for short-term memory for sensitization. U0126 (Active; $n=6$ ) or the inactive analog U0124 (Inactive; $n=5$ ) was applied 70 min before training and was present throughout testing. Training was performed using a single shock to the tail $(1 \times S)$. Data are expressed as mean \pm SEM duration of T-SW normalized to baseline. Inset, A single pulse of 5-HT does not activate MAPK. Pleural-pedal ganglia were treated with one pulse of 5-HT, SN clusters were excised, and MAPK activation was examined using phosphoMAPK and phospho-independent MAPK antibodies. Sample blots with phospho-MAPK and total MAPK antibodies are shown.

$\left(F_{(2,14)}=4.15, p<0.05 ;\right.$ U0124-trained vs U0126-trained and U0126-nontrained, both $p<0.05$ ) but no difference between the two groups of U0126-treated preparations (NS). These results show that MAPK activity is required for the induction of LTM for sensitization.

\section{Discussion}

The overall goal of our analysis of MAPK and memory was to establish links between changes observed at molecular and synaptic levels of analysis with distinct behavioral phases of memory for sensitization. Toward that end, we established previously in both intact animals and reduced preparations that tail shock serves as an effective reinforcer in inducing sensitization of the T-SW reflex (Sutton et al., 2001, 2002). In addition, tail shock and tail nerve shock (1) both induce a wide range of comparable neural correlates of sensitization at $\mathrm{SN}-\mathrm{MN}$ synapses (Carew et al., 1979; Frost et al., 1985; Bunge et al., 1997; Cleary et al., 1998;

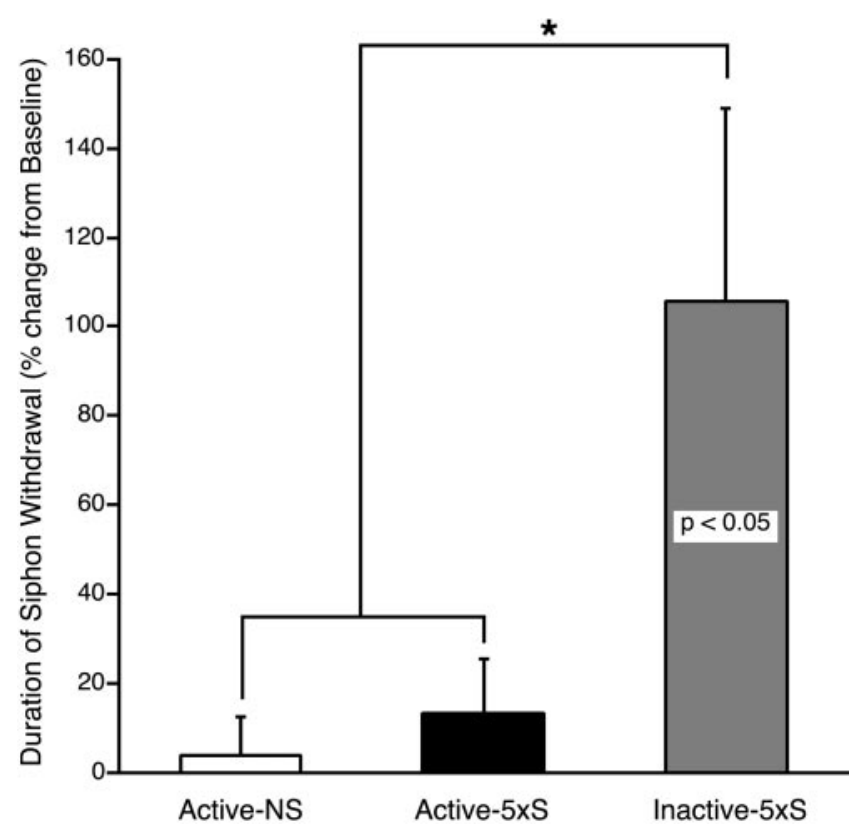

Figure 6. MAP kinase activity is required for long-term memory for sensitization. U0126 (Active) or the inactive analog U0124 (Inactive) was applied to the ring ganglia $30 \mathrm{~min}$ before training and was present during training and for $1 \mathrm{hr}$ after training. Training was performed using five spaced shocks to the tail $(5 \times S)$. NS, Control preparations not given any shock. Data are expressed as mean \pm SEM duration of T-SW (percentage of change from baseline; Active$5 \times S, n=6$; Inactive $5 \times S, n=6$; Active-NS, $n=5$ ). The $p$ value in the histogram reflects a within-group comparison (one tail); ${ }^{*} p<0.05$ denotes significance for between-group comparisons.

Marinesco and Carew, 2002), and (2) both induce the release of 5 -HT at the SN cell bodies and their synaptic terminals (Marinesco and Carew, 2002; Marinesco and Carew, unpublished observations). Thus, tail nerve shock can serve as an effective analog of sensitization training. Moreover, we and several others have also shown that direct application of 5-HT to the CNS, in patterns mimicking behaviorally relevant training with tail shock, also induce the same neural correlates of sensitization as induced by tail shock. Thus, application of 5-HT to both the intact CNS and $\mathrm{SN}-\mathrm{MN}$ in culture also has served as an important analog of sensitization training (Montarolo et al., 1986; Ghirardi et al., 1995; Mauelshagen et al., 1996; Martin et al., 1997; Sutton and 
Carew, 2000). Finally, observations of different forms of synaptic plasticity at the tail $\mathrm{SN}$-tail MN synapses induced by different patterns of 5-HT have directly predicted the existence and mechanisms underlying two unique forms of behavioral intermediateterm memory for sensitization (Sutton et al., 2001; Sutton and Carew, 2003). Thus, although there are certainly important interneuronal linkages between the tail SNs and the siphon MNs that modulate the T-SW reflex (Cleary et al., 1995; Bristol et al., 2000), the tail SN-tail MN synapse has proven to be a powerful predictor of behavioral plasticity in this system (Sutton et al., 2001; Sutton and Carew, 2003). Given these connecting experimental links, in this paper, we use tail shock to induce behavioral memory, tail nerve shock as an analog of sensitization training to induce synaptic plasticity at the tail SN-tail MN synapses, and both tail shock and 5-HT to induce MAPK activation. Collectively, these studies revealed the differential role of MAPK in three distinct phases of memory for sensitization in Aplysia.

\section{Activation of MAP kinase by serotonin and tail shock}

MAP kinase is activated when phosphorylated at threonine and tyrosine residues by the upstream dual kinase MEK. We used an antibody that recognizes phosphorylated MAPK, as well as an antibody that is independent of the phosphorylation state of the protein to examine MAPK activation. Consistent with previous observations (Michael et al., 1998; Purcell et al., 2003), a single pulse of 5-HT had no effect on MAPK activation in the SNs. However, sustained MAPK activation was observed after five spaced pulses of 5-HT. Importantly, five spaced tail shocks also induced sustained MAPK activation in the SNs. Because tail shock as well as tail nerve shock induce the release of 5-HT (Marinesco and Carew, 2002; Marinesco and Carew, unpublished observations), the observation that repeated tail shocks also induce MAPK activation with a qualitatively similar time course as repeated 5-HT pulses provides direct links between the molecular analysis of MAPK activation and our subsequent behavioral experiments using tail shock to induce behavioral memory (see below). We should emphasize that tail shock, as well as tail nerve shock, likely also induce the release of other modulatory molecules in the CNS in addition to 5-HT. However, the fact that tail shock and tail nerve shock induce several neural correlates of sensitization and exogenous 5-HT reliably mimics these correlates strongly supports the general model that tail shock-induced 5-HT release provides at least one key modulatory input that plays an important role in MAPK activation and in the induction of sensitization.

The fact that five spaced pulses of 5-HT induce MAPK activation, whereas a single pulse does not, is similar to the observation that MAPK is activated in the hippocampus after multi-trial but not a single trial training in the Morris water maze (Blum et al., 1999). Sustained activation of MAPK has also been observed in the dentate gyrus by BDNF (Ying et al., 2002), in cortical neurons by carbachol (Rosenblum et al., 2000), and after learning (Swank and Sweatt, 2001). The mechanism of 5-HT-induced MAPK activation in Aplysia is not yet known. It has been proposed that protein kinase A, which is activated by 5-HT in the SNs (Müller and Carew, 1998), and/or the release of growth factor(s) may contribute to MAPK activation (Martin et al., 1997; Michael et al., 1998; Purcell et al., 2003).

\section{MAP kinase, synaptic facilitation, and memory}

We showed that inhibiting MAPK inhibits ITF at tail SN-tail MN synapses. In addition, we showed that MAPK activity is required for ITM and LTM but not for STM for sensitization. These re- sults, together with previous observations of Martin et al. (1997) that MAPK activity is required for long-term but not short-term facilitation of cultured SN-MN synapses, suggest that MAPK activity is required specifically for long-lasting forms of synaptic facilitation and memory. In addition, we found that MAPK activity is required for the induction but not expression of ITM, because the MEK inhibitor blocked ITM when applied only during training but had no effect after ITM was established. This is in agreement with recent reports demonstrating that MAPK activity is required for the induction but not maintenance of long-term potentiation (Ying et al., 2002) and long-term memory (Berman et al., 1998). The significance of sustained MAPK activation is not known at present. It is possible that sustained MAPK activation is required for the formation of LTM, but it is also possible that it may simply take time for the MAPK activity to return to basal levels. It has been shown that blocking MAPK activation after training leads to impairment of long-term memory (Blum et al., 1999; Walz et al., 1999). Thus, MAPK activity may be required for some time after training for the consolidation of LTM.

It is interesting that, although MAPK activity is not required for STM induced by one tail shock, memory at 15 min after the last of five spaced tail shocks (which might be considered STM) was virtually abolished when MAPK was inhibited. This suggests that the memory at $15 \mathrm{~min}$ after five spaced tail shocks may reflect the onset of ITM rather than STM. In fact, recent evidence supports this view: memory at $5 \mathrm{~min}$ after one tail shock is not susceptible to protein synthesis inhibitor, whereas memory at the same time point after five spaced tail shocks is (Sutton et al., 2001). Collectively, the results suggest that the memory expressed at 15 min after five training trials is not STM but rather the beginning of the ITM process.

How do our observations in Aplysia relate to the role of MAPK in synaptic plasticity and memory in other systems? Growing evidence implicates activation of the MAPK cascade as a critical step in establishing LTP and memory (for review, see Orban et al., 1999; Thiels and Klann, 2001; Adams and Sweatt, 2002). Thus, MAPK emerges as a highly conserved molecular step in the formation of long-term memories in a variety of systems. Furthermore, our results provide evidence that MAPK plays important roles in intermediate-term memory as well.

\section{Molecular targets of MAP kinase in synaptic facilitation and memory}

MAPK targets seem to be both nuclear as well as cytoplasmic (Bailey et al., 1997; Impey et al., 1998; Grewal et al., 1999). The molecular substrates of MAPK involved in the intermediate-term facilitation and memory are not yet known. Because ITF and ITM require translation but not transcription (Ghirardi et al., 1995; Sutton and Carew 2000; Sutton et al., 2001), it is possible that MAPK may regulate protein synthesis required for the induction of ITF and ITM. Consistent with this hypothesis, Takei et al. (2001) showed recently that BDNF-induced protein synthesis in cortical neurons requires MAPK activity.

The role of MAPK in the induction of LTF and LTM is likely to involve the regulation of downstream transcription factors (and possibly cytoplasmic substrates) (Bailey et al., 1997). Among the transcription factors, cAMP response element-binding protein (CREB), CCAAT/enhancer-binding protein (C/EBP), and Elk-1 are all potential candidates. Phosphorylation of CREB and consequent cAMP response element (CRE)-dependent gene expression is essential for establishing persistent synaptic plasticity and memory (Dash et al., 1990; Alberini et al., 1995; Silva et al., 1998). In Aplysia, CREB2 and C/EBP have been shown to be phosphor- 
ylated by MAPK (Michael et al., 1998), and MAPK phosphorylation of C/EBP increases its DNA binding activity (Yamamoto et al., 1999). CREB2 is a repressor of CREB1a (Bartsch et al., 1995), and phosphorylation of CREB2 by MAPK in principle could lead to the de-repression of CREB1a, enabling CREB-mediated gene induction required for the LTF and LTM (Martin et al., 1997; Michael et al., 1998). In addition, CREB phosphorylation can also be regulated by MAPK through p90rsk, the intermediary kinase between MAPK and CREB (Xing et al., 1996; Impey et al., 1998; Roberson et al., 1999; Vanhoutte et al., 1999; Davis et al., 2000). Phosphorylation of Elk-1, a MAPK substrate, has been observed after LTP induction (Davis et al., 2000) and learning (Berman et al., 1998; Cammarota et al., 2000). In complex with the serum response factor, phosphorylated Elk-1 plays important roles in serum-response element (SRE)-dependent transcription (Wasylyk et al., 1998). SREs are present in the promoter region of several immediate early genes, including those that are induced during activity-dependent synaptic plasticity (Abraham et al., 1993; Worley et al., 1993). Thus, the pivotal role played by MAPK in intermediate- and long-term phases of plasticity and memory may relate to its versatility in translational and transcriptional regulation.

\section{References}

Abraham WC, Demmer J, Richardson LC, Williams JM, Lawlor PA, Mason SE, Tate WP, Dragunow M (1993) Correlations between early gene induction and the persistence of LTP. Neuroscience 56:717-727.

Adams JP, Sweatt JD (2002) Molecular psychology: roles for the ERK MAP kinase cascade in memory. Annu Rev Pharmacol Toxicol 42:135-163.

Alberini CM, Ghirardi M, Huang YY, Nguyen PV, Kandel ER (1995) A molecular switch for the consolidation of long-term memory: cAMPinducible gene expression. Ann NY Acad Sci 758:261-285.

Atkins CM, Selcher JC, Petraitis JJ, Trzaskos JM, Sweatt JD (1998) The MAPK cascade is required for mammalian associative learning. Nat Neurosci 1:602-609.

Bailey CH, Kang BK, Chen M, Martin KC, Lim CS, Casadio A, Kandel ER (1997) Mutation in the phosphorylation sites of MAP kinase blocks learning-related internalization of apCAM in Aplysia sensory neurons. Neuron 18:913-924.

Bartsch D, Ghirardi M, Skehel PA, Karl KA, Herder SP, Chen M, Bailey CH (1995) Aplysia CREB2 represses long-term facilitation: relief of repression converts transient facilitation into long-term functional and structural change. Cell 83:979-992.

Berman DE, Hazvi S, Rosenblum K, Seger R, Dudai Y (1998) Specific and differential activation of mitogen-activated protein kinase cascades by unfamiliar taste in the insular cortex of the behaving rat. J Neurosci 18:10037-10044.

Blum S, Moore AN, Adams F, Dash PK (1999) A mitogen-activated protein kinase cascade in the CA1/CA2 subfield of the dorsal hippocampus is essential for long-term spatial memory. J Neurosci 19:3535-3544.

Bristol AS, Sutton MA, Carew TJ (2000) Functional architecture of the neural circuit underlying tail-elicited siphon withdrawal in Aplysia. Soc Neurosci Abstr 26:568.7.

Bunge SA, Mauelshagen J, Carew TJ (1997) Reversal of relative thresholds for synaptic facilitation and increased excitability induced by serotonin and tail nerve stimulation in Aplysia sensory neurons. Neurobiol Learn Mem 67:259-263.

Cammarota M, Bevilaqua LRM, Ardenghi P, Paratcha G, Levi de Stein M, Izquierdo I, Medina JH (2000) Learning-associated activation of nuclear MAPK, CREB and Elk-1, along with Fos production, in the rat hippocampus after a one-trial avoidance learning: abolition by NMDA receptor blockade. Mol Brain Res 76:36-46.

Carew T, Castellucci VF, Kandel ER (1979) Sensitization in Aplysia: restoration of transmission in synapses inactivated by long-term habituation. Science 205:417-419.

Castellucci VF, Blumenfeld H, Goelet P, Kandel ER (1989) Inhibitor of protein synthesis blocks long-term behavioral sensitization in the isolated gill-withdrawal reflex of Aplysia. J Neurobiol 20:1-9.

Chin J, Angers A, Cleary LJ, Eskin A, Byrne JH (2002) Transforming growth factor beta1 alters synapsin distribution and modulates synaptic depression in Aplysia. J Neurosci 22:RC220(1-6).

Cleary LJ, Byrne JH, Frost WN (1995) Role of interneurons in defensive withdrawal reflexes in Aplysia. Learn Mem 2:133-151.

Cleary LJ, Lee WL, Byrne JH (1998) Cellular correlates of long-term sensitization in Aplysia. J Neurosci 18:5988-5998.

Crow T, Siddiqi V, Dash PK (1997) Long-term enhancement but not shortterm in Hermissenda is dependent upon mRNA synthesis. Neurobiol Learn Mem 68:343-350.

Crow T, Xue-Bian, J-J, Siddiqi, V Kang T, Heavy JT (1998) Phosphorylation of mitogen-activated protein kinase by one-trial and multi-trial classical conditioning. J Neurosci 18:3480-3487.

Dash PK, Hochner B, Kandel ER (1990) Injection of the cAMP-responsive element into the nucleus of Aplysia sensory neurons blocks long-term facilitation. Nature 345:718-721.

Davis S, Vanhoutte P, Pagès C, Caboche J, Laroche S (2000) The MAPK/ MAPK cascade targets both Elk-1, cAMP response element-binding protein to control long-term potentiation-dependent gene expression in the dentate gyrus in vivo. J Neurosci 20:4563-4572.

DeSilva DR, Jones EA, Favata MF, Jafee BD, Magdela RL, Trzaskos JM, Scherle PA (1998) Inhibition of mitogen activated protein kinase kinase blocks T-cell proliferation but does not induce or prevent anergy. J Immunol 160:4175-4181.

DeZazzo J, Tully T (1995) Dissection of memory formation: from behavioral pharmacology to molecular genetics. Trends Neurosci 18:212-218.

English JD, Sweatt JD (1997) A requirement for the mitogen-activated protein kinase cascade in hippocampal long-term potentiation. J Biol Chem 272:19103-19106.

Frost WN, Castellucci VF, Hawkins RD, Kandel ER (1985) Monosynaptic connections made by the sensory neurons of the gill- and siphonwithdrawal reflex in Aplysia participate in the storage of long-term memory for sensitization. Proc Natl Acad Sci USA 82:8266-8269.

Ghirardi M, Montarolo PG, Kandel ER (1995) A novel intermediate stage in the transition between short- and long-term facilitation in the sensory to motor neuron synapse of Aplysia. Neuron 14:413-420.

Goldsmith JR, Byrne JH (1993) Bag cell extract inhibits tail-siphon withdrawal reflex, suppresses long-term but not short-term sensitization, and attenuates sensory-to-motor neuron synapses in Aplysia. J Neurosci 13:1688-1700.

Grewal SS, York RD, Stork PJ (1999) Extracellular signal-regulated kinase signalling in neurons. Curr Opin Neurobiol 9:544-553.

Impey S, Obrietan K, Wong ST, Poser S, Yano S, Wayman G, Deloulme JC, Chan G, Storm DR (1998) Cross talk between MAPK and PKA is required for $\mathrm{Ca}^{2+}$ stimulation of CREB-dependent transcription and MAPK nuclear translocation. Neuron 21:869-883.

Laemmli UK (1970) Cleavage of structural proteins during the assembly of the head of bacteriophage T4. Nature 227:680-685.

Levenson J, Byrne JH, Eskin A (1999) Levels of serotonin in the hemolymph of Aplysia are modulated by light/dark cycles and sensitization training. J Neurosci 19:8094-8103.

Levenson J, Endo S, Kategaya LS, Fernandez RI, Brabham DG, Chin J, Byrne JH, Eskin A (2000) Long-term regulation of neuronal high-affinity glutamate and glutamine uptake in Aplysia. Proc Natl Acad Sci USA 97:12858-12863.

Marinesco S, Carew TJ (2002) Serotonin release evoked by tail nerve stimulation in the CNS of Aplysia: characterization and relationship to heterosynaptic plasticity. J Neurosci 22:2299-2312.

Martin KC, Michael D, Rose JC, Barad M, Casadio A, Zhu H, Kandel ER (1997) MAP kinase tranlocates into the nucleus of the presynaptic cell and is required for long-term facilitation in Aplysia. Neuron 18:899-912.

Mauelshagen J, Parker GR, Carew TJ (1996) Dynamics of induction and expression of long-term synaptic facilitation in Aplysia. J Neurosci 16:7099-7108.

Mazzucchelli C, Vantaggiato C, Ciamei A, Fasano S, Pakhotin P, Krezel W, Welzl H, Wolfer DP, Pagès G, Valverde O, Marowsky A, Porrazzo A, Orban PC, Maldonado R, Ehrengruber MU, Cestari V, Lipp H-P, Chapman PF, Pouysségur J, Brambilla R (2002) Knockout of ERK1 MAP kinase enhances synaptic plasticity in the striatum and facilitates striatalmediated learning and memory. Neuron 34:807-820.

Menzel R (2001) Searching for the memory trace in a mini-brain, the honeybee. Learn Mem 8:53-62.

Michael D, Martin KC, Seger R, Ning MM, Baston R, Kandel ER (1998) 
Repeated pulses of serotonin required for long-term facilitation activate mitogen-activated protein kinase in sensory neurons of Aplysia. Proc Natl Acad Sci USA 95:1864-1869.

Montarolo PG, Goelet P, Castellucci VF, Morgan J, Kandel ER, Schacher S (1986) A critical period for macromolecular synthesis in long-term heterosynaptic facilitation in Aplysia. Science 234:1249-1254.

Müller U, Carew TJ (1998) Serotonin induces temporally and mechanistically distinct phases of persistent PKA activity in Aplysia sensory neurons. Neuron 21:1423-1434.

Orban PC, Chapman PF, Brambilla R (1999) Is the Ras-MAPK signalling pathway necessary for long-term memory formation? Trends Neurosci 22:38-44.

Patterson SL, Pittenger C, Morozov A, Martin KC, Scanlin H, Drake C, Kandel ER (2001) Some forms of cAMP-mediated long-lasting potentiation are associated with release of BDNF and nuclear translocation of phospho-MAP kinase. Neuron 32:123-140.

Purcell AL, Sharma SK, Bagnall MW, Sutton MA, Carew TJ (2003) Activation of a tyrosine kinase-MAPK cascade enhances the induction of longterm synaptic facilitation and long-term memory in Aplysia. Neuron $37: 473-484$.

Roberson ED, English JD, Adams JP, Selcher JC, Kondratick C, Sweatt JD (1999) The mitogen-activated protein kinase cascade couples PKA and PKC to cAMP response element binding protein phosphorylation in area CA1 of hippocampus. J Neurosci 19:4337-4348.

Rosenblum K, Futter M, Jones M, Hulme EC, Bliss TV (2000) ERKI/II regulation by the muscarinic acetylcholine receptors in neurons. J Neurosci 20:977-985.

Rosenzweig MR, Bennett EL, Colombo PJ, Lee DW, Serrano PA (1993) Short-term, intermediate-term, and long-term memories. Behav Brain Res 57:193-198.

Schafe GE, Nadel NV, Sullivan GM, Harris A, LeDoux JE (1999) Memory consolidation for contextual and auditory fear conditioning is dependent on protein synthesis, PKA, and MAP kinase. Learn Mem 6:97-110.

Selcher JC, Atkins CM, Trzaskos JM, Paylor R, Sweatt JD (1999) A necessity for MAP kinase activation in mammalian spatial learning. Lean Mem 6:478-490.

Sharma SK, Carew TJ (2002) Inclusion of phosphatase inhibitors during Western blotting enhances signal detection with phospho-specific antibodies. Anal Biochem 307:187-189.

Sharma SK, Sherff CM, Bagnall MW, Sutton MA, Carew TJ (2002) Mitogen-activated protein kinase activity is required for long-lasting forms of synaptic facilitation and memory in Aplysia. Soc Neurosci Abstr 28:552.14.

Silva AJ, Kogan JH, Frankland PW, Kida S (1998) CREB and memory. Annu Rev Neurosci 21:127-148.

Sutton MA, Carew TJ (2000) Parallel molecular pathways mediate expression of distinct forms of intermediate-term facilitation at tail sensorymotor synapses in Aplysia. Neuron 26:219-231.

Sutton MA, Carew TJ (2003) Behavioral, cellular and molecular analysis of memory in Aplysia. I. Intermediate-term memory. Integ Comp Biol 42:725-735.

Sutton MA, Masters SE, Bagnall MW, Carew TJ (2001) Molecular mechanisms underlying a unique intermediate phase of memory in Aplysia. Neuron 31:143-154.
Sutton MA, Ide J, Masters SE, Carew TJ (2002) Interaction between amount and pattern of training in the induction of intermediate- and long-term memory for sensitization in Aplysia. Learn Mem 9:29-40.

Swank MW, Sweatt JD (2001) Increased histone acetyltransferase and lysine acetyltransferase activity and biphasic activation of the ERK/RSK cascade in insular cortex during novel taste learning. J Neurosci 21:3383-3391.

Takei N, Kawamura M, Hara K, Yonezawa K, Nawa H (2001) Brain-derived neurotrophic factor enhances neuronal translation by activating multiple initiation processes. Comparison with the effects of insulin. J Biol Chem 276:42818-42825.

Thiels E, Klann E (2001) Extracellular signal-regulated kinase, synaptic plasticity, and memory. Rev Neurosci 12:327-345.

Vanhoutte P, Barnier JV, Guibert B, Pagès C, Besson MJ, Hipskind RA, Caboche J (1999) Glutamate induces phosphorylation of Elk-1 and CREB, along with c-fos activation, via an extracellular signal-regulated kinasedependent pathway in brain slices. Mol Cell Biol 19:136-146.

Walz R, Rosler R, Quevedo J, Rochenback IC, Amaral OB, Vianna MR, Lenz G, Medina JH, Izquierdo I (1999) Dose-dependent impairment of inhibitory avoidance retention in rats by immediate post-training infusion of a mitogen-activated protein kinase kinase inhibitor into cortical structures. Behav Brain Res 105:219-223.

Walz R, Rosler R, Quevedo J, Sant'Anna MK, Mainhibitora M, Rodrigues C, Gottfried C, Medina JH, Izquierdo I (2000) Time-dependent impairment of inhibitory avoidance retention in rats by posttraining infusion of a mitogen-activated protein kinase kinase inhibitor into cortical and limbic structures. Neurobiol Learn Mem 73:11-20.

Wasylyk B, Hagman J, Gutierrez-Hartmann A (1998) Ets transcription factors: nuclear effectors of the Ras-MAP-kinase signaling pathway. Trends Biochem Sci 23:213-216.

Winder DG, Mansuy IM, Osman M, Moallem TM, Kandel ER (1998) Genetic and pharmacological evidence for a novel, intermediate phase of long-term potentiation suppressed by calcineurin. Cell 92:25-37.

Winder DG, Martin KC, Muzzio IA, Rohrer D, Chruscinski A, Kobilka B, Kandel ER (1999) MAPK plays a regulatory role in induction of LTP by theta frequency stimulation and its modulation by beta-adrenergic receptors. Neuron 24:715-726.

Worley PF, Bhat RV, Baraban JM, Erickson CA, McNaughton BL, Barnes CA (1993) Thresholds for synaptic activation of transcription factors in hippocampus: correlation with long-term enhancement. J Neurosci 13:4776-4786.

Wu SP, Lu KT, Chang WC, Gean PW (1999) Involvement of mitogenactivated protein kinase in hippocampal long-term potentiation. J Biomed Sci 6:409-417.

Xing J, Ginty DD, Greenberg ME (1996) Coupling of the RAS-MAPK pathway to gene activation by RSK2, a growth factor-regulated CREB kinase. Science 273:959-963.

Yamamoto N, Hegde AN, Chain DG, Schwartz JH (1999) Activation and degradation of the transcription factor C/EBP during long-term facilitation in Aplysia. J Neurochem 73:2415-2423.

Ying SW, Futter M, Rosenblum K, Webber MJ, Hunt SP, Bliss TVP, Bramham CR (2002) Brain-derived neurotrophic factor induces long-term potentiation in intact adult hippocampus: requirement for ERK activation coupled to CREB and upregulation of Arc synthesis. J Neurosci 22: 1532-1540. 